\title{
Einige Worte zu meinem Referate über Dr. G. Joseph's Artikel: ,Ueber Fliegen als Schädlinge und Parasiten des Menschen."
}

\section{Von Jos. Mik.}

Herrn Dr. Jo se ph in Breslau hat es beliebt, sich in einer den Entomologen ferner stehenden Fachschrift, nämlich in der "Deutschen Medicinal-Zeitung" (1887, Nr. 5, Berlin), über mein Referat (in der "Wiener Entomol. Ztng." 1886, pag. 238), das seinen obencitirten Artikel zum Gegenstande hat, in einer derartig persönlichen Weise auszulassen, dass ich nicht umhin kann, hierüber an dieser Stelle einige Worte zu sagen, mit der Bemerkung, dass ich auch den Lesern der genannten ärztlichen Fachschrift die nöthige Aufklärung nicht schuldig bleiben werde.

Schon der Zusatz am Titel des Aufsatzes von H. Dr. J.: „Zugleich Zurückweisung der Ausfälle des H. Gymnas.-Prof. Mik gegen meine Abhandlung: „Ueber Fliegen als Schädlinge etc." " lässt die subjective Beeinflussung des Verfassers errathen, und Jeder, der mein Referat gelesen hat, möge urtheilen, ob dasselbe "Ausfälle" enthalte?

Doch hören wir, auf welche Art H. Docent Dr. J. diese „Ausfälle" abweist?

Vor Allem führt er die Autorität "des grössten jetzt lebenden Oestridenkenners", Prof. Brauer's, den er doch in seinem früheren, von mir recensirten Aufsatze gänzlich ignorirt hat*), gegen mein Referat in's Feld. Nach Brauer ist es erwiesen, sagt der Herr Docent, dass Oestridenlarven unter die Haut des Menschen gelangen und hier leben können.

Das habe ich aber in meinem Referate ja gar nicht angezweifelt; mir erschien es nur unglaublich, - und auch heute bin ich noch nicht bekehrt - dass die Larve eines europäischen

*) Man vergleiche mein Referat, worin ich sage, dass verschiedene Thatsachen, welche II. Dr. J. in seiner Arbeit über Hautoestriden (Deutsche Medic. Ztschr. 1885, Nr. 4) "feststellt", längst von B ra ue r festgestellt worden sind. - Man vergleiche aber auch, was B r a u er in der Wien. Entom. Ztng. 1887, pag. 74 sagt: „Ein gewissenhafter Autor oder Compilator hat daher letztere Mittheilung nicht todt zu schweigen und meine Entdeckungen nicht Herrn Goudot zuzuschreiben", eine Mahnung, die sich unzweifelhaft auf dasjenige bezieht, was H. Dr. J. über Dermatobia noxialis Goud. sich zu erklären erlaubt hat (vergl. auch Entomol. Nachricht. 1885, pag. 19, oben).

„Wiener Entomologische Zeitung“ VI. Heft 3. (8. April 1887.) 
Hautoestriden bis zur Verpuppungsreife unter des Menschen Haut verbleiben könne.

Weiter sucht H. Dr. J. die von Brauer ausgesprochene Ansicht, dass Hypodermen ihre Brut an den Menschen unter besonderen Umständen absetzen mögen, durch eine Conclusion zu erhärten, welche wohl auf schwachen Beinen einhergeht: Die Oestridenweibchen sollen "aus Noth zu einem anderen Thiere, selbst zum Menschen im Triebe zur Erhaltung der Art die Zuflucht nehmen, um den mächtigen Drang, ihre Eier abzulegen, endlich zu befriedigen". Woher aber diese Conclusion? H. D. J. fing nach seiner Aussage oft Oestridenweibchen, welche "im Fluge" Schafheerden umschwärmten, im Fangglase aber geängstigt ihre Eier ausspritzten. Um das letztere zu beobachten, bedarf es aber erst keiner Oestridenweibchen; das trächtige Weibchen jeder Fliege, jedes Schmetterlinges wird, im Fangglase oder aufgespiesst, in der Angst die Eier oder Larven absetzen. Das Argument des H. Dr. J. beweist also in unserer Angelegenheit gar nichts; denn ich glaube, dass die Oestridenweibchen, in Angst und Noth versetzt, ihre Brut auch in die Luft fallen lassen werden.

Auch die angeführten Beispiele, dass die Larven des Leberegels und andere Eingeweidewürmer sich in den Menschen verirren, haben hier nichts $\mathrm{zu}$ sagen: sie können wohl einem mit der Lebensgeschichte und mit dem Charakter der Oestridenlarven nicht vollkommen vertrauten Arzte imponiren, einen Dipterologen aber, der behauptet, dass unsere Hautoestriden nicht bis zur vollständigen Entwicklung (selbstverständlich des Larvenzustandes) im Menschen verbleiben, wird das alles kalt lassen.

H. Dr. J. rechnet mir es zum Fehler an, dass ich in meinem Referate den Ausdruck gebrauchte, die Hypodermen könnten am Menschen nicht die "ganze Metamorphose" durchmachen. Jedermann, der meinem Referate Verständniss entgegenbringt, wird wohl wissen, dass die Oestriden nur im Larvenzustande Parasiten sind, und wird daher auch meine Schreibweise richtig deuten. Alles Uebrige wären und sind Nergeleien, welche ich H. Dr. J. sogleich vergelten könnte, wenn ich ihm übel nehmen wollte, dass er in seinem neuesten Aufsatze Eryngium campestre eine "Distelpflanze" nennt. Oder habe ich etwa mein Referat mit dem Dufte der Stilblüthen, 
welche sich in dem früheren Artikel*) des H. Dr. J. entfalten, zu würzen versucht, dass derselbe an meiner Schreibweise sich rächen $\mathrm{zu}$ sollen glaubte?

Auch das ist H. Dr. J. nicht recht, dass ich unsere Hypodermen „specifische Parasiten“ nenne. "Ich sei den Beweis schuldig geblieben, dass die europäischen Hypodermen specifischer sind, als die amerikanischen, wie Dermatobia noxialis Goud., von der es feststeht**), dass deren Larven auch beim Menschen Puppenreife erlangen können." Wo steht es aber geschrieben, dass der Referent in seiner Kritik Beweise erbringen soll, namentlich dann, wenn Beweise längst vorliegen? Muss ich H. Dr. J. erst auf Bra uer's Oestridenmonographie (1863) pag. 269 (letzter Absatz) verweisen, wo er den verlangten Beweis zu suchen hat?

Bis hierher war H. Dr. J. so ziemlich bei der Sache geblieben. Wenn ich trotzdem hierüber $\mathrm{zu}$ sprechen mich bewogen fand, so geschah es, um zu zeigen, wie die Beweisführung aussieht, welche H. Dr. J. für hinreichend gehalten, die in meinem Referate ausgesprochenen Ansichten über seinen ersten Artikel zu entkräften.

Nun aber weiter schwingt H. Dr. J. in einer nicht näher zu bezeichnenden Weise die Geissel gegen meine Person: „Es geht aus der ganzen Art der Mik'schen Besprechung hervor, dass derselbe meine Abhandlung nur ganz oberflächlich gelesen hat. Dass derselbe über eine andere Abhandlung gesprochen hat, ohne auch nur einmal hineingeblickt zu haben

*) Man vergleiche nur „Entomol. Nachricht." 1885, pag. 17, Z. 10 v. u., wo es heisst, dass Fliegenlarven zufällig die zu ihren Arten gehörenden Eier verschlucken; oder ibid. pag. 18, Z. 9, wornach "mehrere nnserer Hausthiere, Edelwild u. a. bei den Menschen selten und von Aerzten in beschränkter Zahl angetroffen worden sind" u. s. w.

**) Ueber dieses Feststehen erwäge man aber, was Brauer, der in Bezug auf die Oestriden gewiss massgebende Gewährsmann, in seiner jüngsten Schrift über Oestriden (Wien. Entom. Ztng. 1887, pag. 72) aufrecht hält: „Nicht einmal bei den viel öfter am Menschen beobachteten Dermatobienlarven ist es bis jetzt constatirt, dass sie bis zu ihrem Abgange von Menschen ertragen wurden." - Doch möge H. Dr. J. auch den Passus beherzigen, welchen wir in derselben Schrift B rauer's (l. c.) finden und welcher wohl ganz speciell H. Dr. J. angeht: „Mittheilungen über ausgewachsene Hypodermenlarven am Menschen und die Entwicklung derselben nach freiwilligem Abgang zu einer bestimmten Fliege, halte ich entweder für eine Verwechslung mit schnell wachsenden Muscarien oder für absolut unwahr." 
- diesen Beweis mache ich mich anheischig mit seiner Erlaubniss demnächst beizubringen. Man kann nicht in allen $Z_{\text {weigen ener }}$ Wissenschaft bewandert sein. Daraus wird Niemandem ein Vorwurf gemacht werden. Aber sich das Amt eines Kritikers gerade in dem Theile anzumassen, in welchem man noch nicht volle Sicherheit besitzt, zeugt von einer nicht geringen Selbstüberhebung. Herr $\mathrm{Mik}$ hat bis jetzt nur der Systematik der Dipteren gehuldigt und nach seinem eigenen Geständniss mit Biologie und Entwicklungsgeschichte sich nicht befasst. Darum in Bezug auf den von mir beschriebenen, biologisch wichtigen Fall kein auf etwa (sic!) angegebenen Gründen basirtes Urtheil, sondern kaum mehr als eine Gefühlsäusserung!"

Vor Allem frage ich H. Dr. J., ob er vielleicht mit einer vierdimensionalen Macht im Bunde stehe, da er von Breslau bis in meine Studirstube zu sehen vermag: da er so sicher weiss, dass ich seine Abbandlung nur oberflächlich gelesen habe? ja - dass ich in eine andere Abhandlung nicht einmal hineingeblickt habe?! Ich freue mich auf den angekündigten Beweis; nur möge der Herr Docent sich einer schlagenderen Beweisführung befleissen, als es in seiner jüngsten Schrift, über die ich hier spreche, geschehen ist.

Der Vorwurf, dass ich mir das Kritikeramt angemasst habe in einer Sache, der ich nicht gewachsen bin, verwandelt sich wohl in einen Vorwurf gegen Herrn Dr. J., nämlich in den, dass es ihm an der nöthigen Literaturkenntniss fehlt; denn sonst hätte er sich zu solch unberechtigtem Anwurfe gegen mich nicht hinreissen lassen können. Oder ist dieser Anwurf etwa nicht mehr als eine blosse "Gefühlsäusserung" von Seiten Herrn Dr. J.'s? Im anderen Falle verweise ich den Herrn Doctor auf die 5 Jahrgänge der "Wiener Entom. Zeitng.". Hier kann er in meine Publicationen über Biologie und Entwicklungsgeschichte der Insecten Einsicht nehmen: es ist kein Jahrgang, in dem er solche nicht finden möchte. Auch in den Schriften der k. k. Zoolog.-Botan. Gesellschaft zu Wien kann er manche meiner Forschungen kennen lernen, die so beiläufig das Gebiet der Biologie betreffen. Nota bene: niemandem ist es aber noch eingefallen, über den Inhalt dieser meiner biologischen Publicationen seinen Unglauben zu äussern. 
Es mag sein, dass sich H. Dr. J. durch einen von mir an ihn gerichteten Brief verleiten liess, ein voreiliges Urtheil gegen mich auszusprechen. Ich muss dann aber doch erwähnen, dass es nicht recht schicklich ist, Privatbriefe $\mathrm{zu}$ gewissen "Beschneidungen" des Wissens des Briefschreibers in der Oeffentlichkeit auszunützen! H. Dr. J. sandte mir ohne weiters einmal zwei ziemlich jämmerlich conservirte Dipterenlarven, in einem Federkiele eingeschlossen, mit dem Ersuchen, dieselben zu bestimmen. Jedermann, der sich mit solchen Dingen beschäftigt, weiss, dass es in vielen Fällen nicht leicht ist, nach solchen Präparaten eine sichere Auskunft zu ertheilen. Es wurde die Gruppe angegeben, in welche die Larven gehören, mit dem Bedeuten, die den Sinn hatte - an den Wortlaut erinnere ich mich nicht mehr - , dass ich mich viel zu wenig mit Biologie abgebe, als dass ich eine genaue Bestimmung der fraglichen Larven vornehmen könnte. Dieses Bedeuten begleitete das Ansuchen, meine Bestimmung nicht etwa zu einer Publication zu benützen, da ich eben keine volle Gewissheit in dieser (speciell vorliegenden) Sache habe. Wie H. Dr. J. dieses Ergebniss ausgenützt, haben wir in seiner jüngsten, gegen mich gerichteten Schrift gesehen.

Ein nächstes Mal, wenn H. Dr. J. wieder etwas Entomologisch-Biologisches schreibt, werde ich nicht unterlassen, ihn um Erlaubniss zu fragen, ob ich diese seine Schrift einer Kritik unterziehen darf oder nicht?

Wenn ich in meinem Referate meine Ansichten über des Herrn Docenten Publication über das Vorkommen der Hautoestriden am Menschen nicht begründet habe, so geschah es, weil ich mich als Referent hierzu durchaus nicht verpflichtet fühlte. Es ist wohl nicht die Aufgabe eines Referenten, seine Ansichten zu begründen, wie ich schon oben sagte. Das sind Forderungen, die man nur anmassenter Weise entweder im Gefühle des Eigendünkels oder in völliger Unwissenheit dessen, was gang und gäbe ist, stellen kann. Jetzt, da ich hierzu herausgefordert worden bin, werde ich eine Begründung meines Urtheiles in puncto der Entwicklung von Hypoderma Diana Br. aus dem Menschen Herrn Dr. J. freilich nicht sebuldig bleiben.

Zuvor aber will ich noch die weiteren Auslassungen des Herrn Docenten gegen meine Person ein wenig beleuchten. Nicht nur, dass er mir die Fähigkeit zu einer Kritik in 
Biologicis abgesprochen, so wendet sich H. Dr. J. jetzt auch gegen mein Urtheil in Systematicis.

Allerdings muss ich ihm für die folgende freundliche Aeusserung meinen verbindlichsten Dank aussprechen: „Sicher fühlt sich Mik auf dem systematischen Gebiete."

Was folgt aber weiter? - Es heisst:

„Da sitzt er scharf zu Gericht und nennt die von mir nach dem Vorgange Portschinsky's adoptirte Bezeichnung der aus den (der Myiasis muscosa zum Grunde gelegenen) Larven von mir erzogenen Fliege mit Sarcophila Wohlfahrti P. geradezu unrichtig. Ich hätte dieselbe mit dem freilich älteren Namen Sarc. magnifica Schin., mit der sie allerdings grosse Aehnlichkeit hat, bezeichnen sollen! Da ich aber von der Artidentität beider noch nicht überzeugt bin, so halte ich deren Confundirung für mindestens voreilig und ungerechtfertigt. - Ich habe mehrfach die Fliege erzogen, aber dieselbe nie im Freien beobachtet. Schiner sagt von seiner Sarc. magnifica, dass sie sich an Eryngium campestre finde. In der Nähe des Oderufers und an vielen anderen Orten um Breslau wächst und blüht diese Distelpflanze vom Juli bis in den August sehr häufig. Obgleich sowohl ich selbst, als auch im Sehen geübte Freunde und meine Söhne durch länger als ein Jahrzehnt blühende Eryngien eifrig und aufmerksam abgesucht haben, so war doch unsere Mühe bisher eine vergebliche. Es gelang uns nicht, im Freien die bezeichnete Fliege aufzufinden. Demnach scheint S. magnifica Sch. um Breslau nicht vorzukommen. Aber selbst wenn es gelungen wäre, S. magnifica aufzufinden, würde ich von der Zusammengehörigkeit beider zu einer Art nur dann überzeugt sein, wenn es gelingen würde, auch S. magnifica aus der Larve aufzuziehen oder wenigstens eine aus ihren Eiern (sic!) geschlüpfte Larve mit der der S. Wohlfahrti als übereinstimmend zu erkennen. Vollkommen gleiche Entwicklung, gleiche frühere Zustände haben zur Feststellung der Artidentität mehr Werth als gleiche Gestalt der ausgebildeten Fliegen. Aus Vorstehendem ergibt sich, dass Mik im Drange, meine Arbeit fehlerhaft zu finden, höchst willkürlich geurtheilt hat. Man hätte erwarten sollen, dass ein Systematiker vom Schlage des Herrn Mik viel peinlicher in dem Urtheile über Zusammengehörigkeit zweier Arten sein sollte. Durch 
ein falsches Urtheil hat $\mathrm{Mik}$ ein ungünstiges Urtheil über seine eigenen Principien der Unterscheidung gesprochen."

Zuerst Lob, dann aber - nach vorausgegangenem langen Gesalbader - Tadel von Seiten des Herrn Dr. J., als wäre er der competenteste Richter auf systematischem Gebiete (man vergl. seine Publication über Grottenkäfer). - Das muss man sich eben gefallen lassen!

Dennoch aber ist und bleibt Sarcophila Wohlfahrti Portsch. synonym mit S. magnifica Schin., und etwas anderes habe ich auch in meinem Referate nicht behauptet. Es ist und bleibt also auch unrichtig, den Namen $S$. Wohlfahrti Portsch. für eine eigene Art zu verwenden. Dies wird Jeder zugeben, der mit den Nomenclaturgesetzen nur halbwegs vertraut ist.

Ein anderes Bewandtniss hat es freilich mit der famosen Logik des Herrn Dr. J. - Weil er und seine im Sehen geübten Freunde Sarc. magnifica auf Eryngium in der Breslauer Gegend nicht angetroffen haben, darum also muss diese Fliege in der dortigen Gegend fehlen?! Es wird viele Dipterenarten geben, welche der Breslauer Fauna angehören, welche ferner gewisse Pflanzen mit Vorliebe besuchen, und dennoch wird sie H. Dr. J. sammt seinen im Sehen geïbten Freunden, auch wenn er sehr fleissig um Breslau Fliegen fängt, nicht auffinden!

Ich aber besitze hinwiederum gegen ein Dutzend von Sarc. magnifica in meiner Sammlung, welche ich alle selbst gefangen habe, - aber ja nicht auf Eryngium! Mit Blüthenthieren geht es einem eben schon nicht anders. Hat er denn aber schon die sogenannte Sarcoph. Wohlfahrti Portsch. um Breslau gefangen, deren Larven er alldort doch in den Nasen der Menschen angetroffen?

Und was wird H. Dr. J. antworten, wenn ich ihn um die Unterschiede frage, die er zwischen seinen gezogenen Thieren (nämlich zwischen seiner S. Wohlfahrti) und zwischen der Beschreibung von S. magnifica herausgefunden hat?

Uebrigens war ja die Auslassung über die richtige Benennung der Sarcophila-Art in meinem Referate nur eine Nebensache; das Hauptgewicht legte ich auf die Zucht von Hypoderma Diana aus dem Menschen. Hic Rhodus, hic salta!

Wie ich also in einer solchen nebensächlichen, doch aber sachlich richtigen Bemerkung den „Drang“ zu erkennen gegeben haben soll, die Arbeit des H. Dr. J. fehlerhaft zu finden, 
ist mir nicht recht klar. Möge es mir H. Dr. J. nicht übel nehmen, wenn ich hier mittheile, dass mir derselbe völlig gleichgiltig war und ist. Um die Sache aber war es mir zu thun!

Auch meine Aeusserung über Sarcophaga carnaria war Herrn Dr. J. ein Dorn im Auge. Wenn ich in meinem Referate daran erinnere, dass es mehrere Sarcophaga-Arten gibt, „welche der S. carnaria höchst ähnlich sind, deren Lebensweise übereinstimmend ist und deren Larven noch keineswegs so bekannt sind, dass man aus ihnen auf die Species Schlüsse führen kann", so bin ich von der Wahrheit wohl nicht haarbreit abgewichen. Dennoch erlaubt sich H. Dr. J. über diese Aeusserung rundweg zu sagen: „das streirt wieder in's Gebiet der Biologie und darum ist der Kritiker wieder auf falschem Wege"“, und nochmals kommt er auf die Larven im Federkiele zurück mit den Worten: "dass dieselben Herrn Mik nicht bekannt sind, davon habe ich Beweise in Händen".

Folgt noch eine Glorification von Seiten des H. Dr. J. seiner selbst mit folgenden Worten: "Andere aber, denen seit einem Vierteljahrhundert daran liegt, die Larven gerade dieser häufigen und einander so ähnlichen Fliegen, welche förmlich erfinderisch sind, um ihre lebendig geborene Brut an animalische Stoffe abzusetzen, in ihrem morphologischen und biologischen Verhalten - behufs Feststellung ärztlich-prophylactischer Massregeln - genau zu unterscheiden, möchten in diesem Punkte etwas besser daran sein. "*)

Hiermit endet die "Zurückweisung" meiner "Ausfälle“.

Bevor ich zur Begründung meines in dem obengenannten Referate abgegebenen Urtheiles über die genannte Arbeit des H. Dr. J. schreite, muss ich noch einer Fussnote erwähnen, welche gleich auf der ersten Seite des jüngsten Artikels des H. Dr. J. die geehrte Redaction der "Deutsch. Medic. Zeitng." (vielleicht?) als Einbegleitung zu diesem Artikel zu geben sich bemüssigt fand. Die Note lautet: "Diese Kritik“ (d. i. ein Abzug meines Referates in der "Wien. Entom. Ztg. ") „wurde vor einiger Zeit auffallenderweise nicht dem Verfasser" (d. i. H.

*) Ob H. Dr. J. um gar so viel besser daran ist, weiss ich nicht: trotz seines ein Vierteljahrhundert dauernden Studiums weiss er noch heute nicht, dass die Sarcophilen larvipar und nicht, wie er behauptet, ovipar sind. (Vergl. oben, pag. 92.) 
Dr. J.), „dessen Arbeit dieselbe zum Gegenstande hat, sondern der Redaction von H. Prof. Mik zugesandt".

Ich weiss nicht, ob jemand Anderer in diesem Vorgange etwas so Auffallendes finden mag? Man pflegt Referate dem betreffenden Verleger oder der Redaction immerhin einzusenden. Ich frage aber auch: woher weiss die geehrte Redaction, dass ich dem Verfasser diesen Abdruck nicht übermittelt habe? Und wenn es nicht geschehen wäre, wem wird das auffallen, wem habe ich hierüber Rechenschaft zu geben?

In Bezug auf die Gründe, welche meinem Misstrauen gegen Herrn Dr. Joseph's Entdeckung, von ausgewachsenen Hypodermenlarven am Menschen Nahrung gegeben, kann ich mich im Folgenden kurz fassen.

Vor Allem muss ich nochmals darauf hinweisen, dass sowohl Hypoderma bovis als auch H. Diana specifische Parasiten sind: jede Art hat bekanntlich ihre ganz bestimmten Thiere, in welchen sie ihre Metamorphose als Parasit durchmacht. Das ist nicht meine Erfindung, das ist oder war wenigstens bis zu Dr. Joseph's Entdeckung eine ausgemachte Thatsache.

Wird nun ein oder der andere Fall zur Kenntniss gebracht, der von dieser Thatsache eine höchst auffallende Ausnahme macht, so müssen alle Umstände, welche die Schilderung dieses ungewöhnlichen Falles begleiten, über allen $\mathrm{Z}$ weifel erhaben sein. Nur dann wird man diesem Falle den "nöthigen Glauben" schenken können.

Insbesondere waren es zwei Gründe, welche mich zu dem ausgesprochenen $Z_{w}$ eifel in meinem Referate führten. Aus den Ausführungen des H. Dr. J. über den Fall, welcher sich auf Hypoderma Diana bezieht, geht hervor, dass die mit der Brut dieser Fliege beschenkte Person "von zwei Aerzten nacheinander mit Quecksilber- und Jodpräparaten behandelt wurde". Wer nun einerseits die Wirkung dieser Präparate auf den thierischen Organismus, anderseits aber die Empfindlichkeit der Hypodermen-Larven in's Auge fasst, dem werden nicht

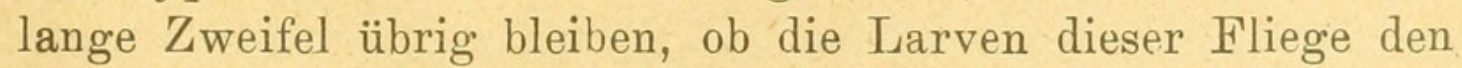
angewandten Curversuchen länger widerstehen konnten?!

Ein anderer Grund meines Misstrauens bezieht sich auf den zweiten von H. Dr. J. mitgetheilten Fall, nämlich von Hypoderma bovis Deg. am Menschen. Es wird hier von einer 
Dasselbeule im Nacken eines Hirten gesprochen, aus welcher sämmtliche, also mehrere Larven (wie viele wird nicht gesagt, was doch äusserst wichtig wäre) extrahirt worden seien. Bekanntlich aber lebt in ein er Dasselbeule stets nur e in e Larve, selbst wenn auch mehrere Larven sich noch so nahe aneinander angesiedelt hätten. Der Umstand schien mir um so massgebender zu sein, als die Beobachtung von einem Arzte gemacht wurde, der wohl zwischen einer gemeinsamen Geschwulst und zwischen mehreren Dasselbeulen einen Unterschied ziehen zu müssen hätte verstehen sollen.

Aber noch ein innerer Grund hat mich in meinem Urtheile über H. Dr. Joseph's Entdeckung bestärkt. Es muss uns Wunder nehmen, dass H. Dr. J. einen pathologisch und entomologisch so wichtigen Fall, wie jenen, den er im Jahre 1864 bezüglich Hypoderma Diana am Menschen entdeckt haben will, mehr als 20 Jahre unveröffentlicht hatte belassen mögen.

So stand die Sache im Juli 1886, als ich mein so übel aufgenommenes Referat publicirte.

Mittlerweile sind zwei Schriften über Oestriden erschienen, welche wohl deutlich genug dafür sprechen, dass meine Ansichten über die vollkommene Entwicklung der Hypodermenlarven im Menschen denn doch berücksichtigungswürdig sind. H. Dr. J. hat freilich zur Zeit, als er seine freundliche „Zurückweisung" meiner "Ausfälle" verfasste, von diesen Schriften noch keine Kenntniss nehmen können.

Die eine derselben hat gerade Prof. Dr. Brauer zum Verfasser, also denjenigen competenten Gewährsmann, welchem H. Dr. J. in seiner "Zurückweisung", wie ich oben angeführt habe, mit Recht den vollsten Glauben entgegenbringt. Wir finden diese Schrift in der "Wiener Entom. Zeitng." 1887, pag. 71, unter dem Titel: „Nachträge zur Monographie der Oestriden" und lesen darin (pag. 72) Folgendes: "Ich möchte allen Jenen, welchen solche Fälle" (Oestridenlarven am Menschen) "vorkommen, empfehlen, meine Ansichten wohl zu erwägen und, wenn solche Nachrichten veröffentlicht werden, zuerst die Verlässlichkeit des Autors zu prüfen", und auf derselben Seite weiter unten: „Mittheilungen über ausgewachsene Hypodermenlarven am Menschen und die Entwicklung derselben nach freiwilligem Abgange zu einer bestimmten 
Fliege, halte ich entweder für eine Verwechslung mit schnellwachsenden Muscarien oder für absolut unwahr". Dass diese Worte besonders an die Adresse des H. Dr. J. gerichtet sind, habe ich bereits oben gesagt.

Eine zweite wichtige Arbeit hat Herr W. M. Schöyen, Conservator am zoolog. Museum der Universität in Christiania, in der "Entomol. Tidskrift af Spångberg“, 1886, Heft 3-4, pag. $171 \mathrm{ff}$., unter dem Titel : „Om Forekomsten af Dipterlarver under Huden hos Mennesker"*) geliefert.

H. Sch öyen theilt uns in dieser Arbeit mit, dass das Vorkommen von Hypodermenlarven am Menschen in Norwegen schon über 1 Jahrhundert lang bekannt sei. Nie aber bleibt die Larve bis zu ihrer vollständigen Entwicklung am Menschen. Die Zeit des Abganges fällt in ihr erstes Stadium oder höchstens zu Beginn des 2. Stadiums, und zwar immer nach einer Wanderung der Larve unter der Haut von den unteren Körpertheilen des Menschen nach den oberen. ${ }^{* *}$ ) Diese Fälle sind verhältnissmässig so häufig, dass sie im Allgemeinen der Bevölkerung bekannt sind. Die Ursache des frühzeitigen $\mathrm{Ab}$ ganges der Larven und der sonderbaren Wanderung unter der Haut ist nach Schöyen das Unbehagen, welches der Mensch als zufälliger Wirth den Hypodermenlarven bereitet.

Pag. 185 heisst es in dieser Arbeit wörtlich über die 2 von Dr. Joseph entdeckten Fälle: "Während nämlich in allen übrigen bekannten Fällen diese Art von Larven im unentwickelten Zustande vertrieben wurde und daher zu Grunde ging, wird erzählt (von Dr. J.), dass sie hier beidemale ihre volle Entwicklung erreichte und in dem einen Falle nach der Verpuppung sogar Bremsen geliefert hat! ****)

Und weiter pag. 186: „Wenn man beachtet, dass der Verfasser (Dr. J.) selbst sagt, dass dies die 2 einzigen Fälle im Laufe eines Vierteljahrhunderts sind, welche Aufmerksamkeit

*) „Ueber das Vorkommen von Dipterenlarven unter der Haut des Menschen."

**) Auch Axon erwähnt wandernder Dipterenlarven unter der Haut des Menschen (vergl. "The Entomologist", 1882, XV, pag. 164) (Mik).

***) Dieses Ausrufungszeichen scheint von H. Sch ö y e n nicht ohne Grund gebraucht worden zu sein. 
verdienen, so muss man wirklich von Glück reden, dass es ihm beidemale gelungen ist, die Hypodermenlarven beim Menschen im Stadium der vollen Entwicklung angetroffen zu haben; denn er ist derjenige, dem es gelungen, dies zu entdecken. Niemandem anderen und nirgends anderswo ist dies geglückt."

An diese letzte Bemerkung schliesst Herr Schöyen aber folgende Note: „Es scheint sehr angezeigt zu sein, zu erwähnen, dass der bekannte Dipterologe Prof. Mik offen erklärt hat, dass er dem Berichte des Dr. Jose ph in Bezug auf diese Fälle nicht den nöthigen Glauben schenkt."

Hierüber möge sich nun jeder Leser sein eigenes Urtheil bilden. Ich aber glaube, dass ich nicht der einzige bin, welcher vorderhand noch auf eine Bestätigung der Entdeckungen des H. Dr. J. wartet!

Wien, am Tage des heil. Joseph (d. i. am 19. März) 1887.

\section{Kleine coleopterologische Mittheilnngen.}

Von Dr. L. von Heyden in Bockenheim.

1. Cneorrhinus Martini $=C n$. Heydeni.

Im Bull. Soc. Ent. France, 1880, pag. XLIII, beschreibt Fairmair e einen Cneorrhinus Martini von Lissabon; derselbe fällt mit Cn. Heydeni Tournier, Annales Soc. Ent. Belg. 1877, pag. 142, zusammen. Letzterer stammt gleichfalls von Lissabon, woher mir Prof. Paulino de Oliveira 2 Exemplare als Martini einsandte. Cn. Heydeni (Martini) hat verwachsene Klauen, während diese bei dem nahe verwandten prodigus $\mathrm{F}$. und ludificator Gyll. deutlich gespalten sind. Ich bin noch nicht sicher, ob der maroccanische prodigus auch im Süden der iberischen Halbinsel vorkommt.

2. Die Gattung Eutheca Baly (Phytophage), Entom. Monthly Mag. 1878, pag. 204, mit der Art Haroldi vom Nyassa-See collidirt mit Eutheca Kiesw. (Dorcatomini) Erichson Ins. Deutschl. 1877, pag. 155 , mit der Art solida von Portugal. Für Eutheca Baly schlage ich vor: Calothe ca. 


\section{$2 \mathrm{BHL}$ Biodiversity Heritage Library}

Mik, Josef. 1887. "Einige Worte zu meinem Referate über Dr. G. JOSEPH's Artikel: "Ueber Fliegen als Schädlinge und Parasiten des Menschen"." Wiener entomologische Zeitung 6, 87-98. https://doi.org/10.5962/bhl.part.17737.

View This Item Online: https://www.biodiversitylibrary.org/item/44060

DOI: https://doi.org/10.5962/bhl.part.17737

Permalink: https://www.biodiversitylibrary.org/partpdf/17737

\section{Holding Institution}

Smithsonian Libraries

\section{Sponsored by}

Smithsonian

\section{Copyright \& Reuse}

Copyright Status: NOT_IN_COPYRIGHT

This document was created from content at the Biodiversity Heritage Library, the world's largest open access digital library for biodiversity literature and archives. Visit BHL at https://www.biodiversitylibrary.org. 